N. Gupta and K. Tahara

Nagoya Math. J.

Vol. 100 (1985), 127-133

\title{
DIMENSION AND LOWER CENTRAL SUBGROUPS OF METABELIAN P-GROUPS
}

\author{
NARAIN GUPTA* AND KEN-ICHI TAHARA \\ To the memory of the late Takehiko Miyata
}

\section{$\S 1$. Introduction}

It is a well-known result due to Sjogren [9] that if $G$ is a finitely generated $p$-group then, for all $n \leqq p-1$, the $(n+2)$-th dimension subgroup $D_{n+2}(G)$ of $G$ coincides with $\gamma_{n+2}(G)$, the $(n+2)$-th term of the lower central series of $G$. This was earlier proved by Moran [5] for $n \leqq p-2$. For $p=2$, Sjogren's result is the best possible as Rips [8] has exhibited a finite 2-group $G$ for which $D_{4}(G) \neq \gamma_{4}(G)$ (see also Tahara $[10,11]$ ). In this note we prove that if $G$ is a finitely generated metabelian $p$-group then, for all $n \leqq p, D_{n+2}^{2}(G) \leqq \gamma_{n+2}(G)$. It follows, in particular, that, for $p$ odd, $D_{n+2}(G)=\gamma_{n+2}(G)$ for all $n \leqq p$ and all metabelian $p$-groups $G$.

\section{§2. Notation and preliminaries}

While the central idea of the proof of our main result stems from Gupta [1], with a slight repetition, it is equally convenient to give a self-contained proof using a less cumbersome notation.

Let $\tilde{\dagger}=Z F(F-1)$ denote the augmentation ideal of the integral group ring $Z F$ of a free group $F$ freely generated by $x_{1}, x_{2}, \cdots, x_{m}, m \geqq 2$. For a fixed prime $p$, let $\left(p^{\alpha_{1}}, p^{\alpha_{2}}, \cdots, p^{\alpha_{m}}\right), \alpha_{1} \geqq \alpha_{2} \geqq \cdots \geqq \alpha_{m}>0$ be an $m$-tuple of $p$-powers, and let $S=\left\langle x_{1}^{p^{\alpha_{1}}}, x_{2}^{p^{\alpha_{2}}}, \cdots, x_{m}^{p^{\alpha_{m}}}, F^{\prime}\right\rangle$ be the normal subgroup of $F$ so that $F / S$ is abelian. Set $\mathcal{Z}=Z F(S-1)$, the ideal of $Z F$ generated by all elements $s-1, s \in S$. For $1 \leqq n \leqq p$, we shall need to investigate the structure of the subgroup $D_{n+2}\left(\tilde{F}_{z}\right)=F \cap\left(1+\mathfrak{f} P+\mathfrak{f}^{n+2}\right)$ of $F$ which consists of all elements $w \in F$ such that $w-1 \in \mathfrak{f} \xi+\mathfrak{f}^{n+2}$. It is clear that $\left[F^{\prime}, S\right] \gamma_{n+2}(F) \subseteq D_{n+2}(\mathfrak{F} \xi)$.

Let $w \in D_{n+2}(\mathfrak{f} \mathfrak{g})$ be an arbitrary element. Then $w-1 \in \mathfrak{f}^{2}$ and it Received July 25, 1984.

*) Research supported by N.S.E.R.C., Canada. 
follows that $w \in F^{\prime}$. Thus, modulo $F^{\prime \prime}$, using the Jacobi identity, we may write $w$ as

$$
w \equiv w_{1} w_{2} \cdots w_{m-1}
$$

where

$$
w_{i}=\prod_{j=i+1}^{m}\left[x_{i}, x_{j}\right]^{d_{i j}}
$$

and $d_{i j}=d_{i j}\left(x_{i}, x_{i+1}, \cdots, x_{m}\right) \in Z F$. For $i=1,2, \cdots, m$, define homomorphisms $\theta_{i}: Z F \rightarrow Z F$ by $x_{k} \rightarrow 1$ if $k \leqq i, x_{k} \rightarrow x_{k}$ if $k>i$. Since the ideals $\mathfrak{f}, \mathfrak{g}$ are invariant under $\theta_{i}$ 's, it follows, using $\theta_{1}, \theta_{2}, \cdots, \theta_{m-2}$ in succession, that if $w-1 \in \mathfrak{f} z+\mathfrak{f}^{n+2}$ then $w_{i}-1 \in \mathfrak{f} \xi+\mathfrak{f}^{n+2}$ for each $i$. For each $k=1,2, \cdots, m$, define

$$
t\left(x_{k}\right)=1+x_{k}+\cdots+x_{k}^{p_{k-1}^{\alpha}} .
$$

Then

$$
\begin{aligned}
t\left(x_{k}\right) & =\sum_{l=1}^{p^{\alpha_{k}}}\left(\begin{array}{c}
p^{\alpha_{k}} \\
l
\end{array}\right)\left(x_{k}-1\right)^{l-1} \\
& \equiv p^{\alpha_{k}}+\left(\begin{array}{c}
p^{\alpha_{k}} \\
p
\end{array}\right)\left(x_{k}-1\right)^{p-1} \bmod \left(p+f^{p}\right) .
\end{aligned}
$$

We can now prove,

LEMMA 2.1. Let $w_{i}$ be as in (2) with $w_{i}-1 \in \mathfrak{f} g+\mathfrak{f}^{n+2}$ and $n \leqq p$. Then, modulo $\xi+\mathfrak{f}^{n}, d_{i j} \equiv t\left(x_{i}\right) a_{i j} \equiv t\left(x_{j}\right) b_{i j}$, where $t\left(x_{i}\right), t\left(x_{j}\right)$ are given by (3), $a_{i j} \in Z$ and $b_{i j} \in Z F$. Moreover, if $\alpha_{i}=\alpha$, then $b_{i j} \in Z$.

Proof. Expansion of $w_{i}-1$ shows

$$
\sum_{j=i+1}^{m}\left\{\left(x_{i}-1\right)\left(x_{j}-1\right)-\left(x_{\jmath}-1\right)\left(x_{i}-1\right)\right\} d_{i j} \in \mathfrak{F} \mathfrak{g}+\mathfrak{f}^{n+2} .
$$

Since $f$ is a free right $Z F$-module on $x_{1}-1, x_{2}-1, \cdots, x_{m}-1$, it follows from (5) that, for all $j=i+1, \cdots, m$,

$$
\left(x_{j}-1\right)\left(x_{i}-1\right) d_{i j} \in \mathfrak{F} p+\mathfrak{f}^{n+2},
$$

which yields

$$
\left(x_{i}-1\right) d_{i j} \in \mathfrak{B}+\mathfrak{f}^{n+1}
$$

and, in turn,

$$
d_{i j} \in t\left(x_{i}\right) Z F+\beta+\mathfrak{f}^{n}
$$

where $t\left(x_{i}\right)$ is given by (3). Since $n \leqq p$, (4) induces that, for $k \geqq i$, $t\left(x_{i}\right)\left(x_{k}-1\right) \equiv p^{\alpha_{i}-\alpha_{k}} p^{\alpha_{k}}\left(x_{k}-1\right) \equiv 0 \bmod \left(\mathfrak{g}+\tilde{f}^{n}\right) . \quad$ Thus $(7)$ implies $d_{i j} \equiv$ 
$t\left(x_{i}\right) a_{i j} \bmod \left(z+\tilde{I}^{n}\right)$ with $a_{i j} \in Z$. Substituting in (5) gives

$$
\left(x_{i}-1\right) \sum_{j=i+1}^{m}\left(x_{j}-1\right) d_{i j} \in \mathfrak{f} g+\mathfrak{f}^{n+2} .
$$

and, as before,

$$
\sum_{j=i+1}^{m}\left(x_{j}-1\right) d_{i j} \in \mathfrak{g}+\mathfrak{f}^{n+1} .
$$

Using the homomorphisms $\theta_{i+1}, \cdots, \theta_{m-1}$ in turn, gives

$$
\left(x_{j}-1\right) d_{i j} \in \mathfrak{g}+\mathfrak{f}^{n+1}
$$

for all $j=i+1, \cdots, m$, since $d_{i j} \equiv t\left(x_{i}\right) a_{i j} \bmod \left(\mathfrak{g}+\mathfrak{f}^{n}\right)$ with $a_{i j} \in Z$. Thus

$$
d_{i j} \in t\left(x_{j}\right) Z F+\mathfrak{g}+\mathfrak{f}^{n},
$$

and if $\alpha_{i}=\alpha_{j}$ then, as before, $d_{i j} \equiv t\left(x_{j}\right) b_{i j} \bmod \left(\mathfrak{g}+\mathfrak{f}^{n}\right)$ with $b_{i j} \in Z$. This completes the proof of the lemma.

Now, let $\frac{\partial}{\partial x_{k}} d$ be a free partial derivative of $d \in Z F$ with respect to $x_{k}$. Then we prove,

$$
\begin{aligned}
& \text { LEMMA 2.2. } \frac{\partial}{\partial x_{k}} d_{i j} \in p^{\alpha_{k}} Z F+\mathfrak{g}+\tilde{\mathfrak{f}}^{n-1}, i<k \text {, and } \\
& \qquad \frac{\partial}{\partial x_{i}} d_{i j} \in \begin{cases}p^{\alpha_{i}} Z F+\mathfrak{g}+\mathfrak{f}^{n-1} & \text { if } \alpha_{i}=\alpha_{j} \\
p^{\alpha_{i}} Z F+p^{\alpha_{i}-1}\left(x_{i}-1\right)^{p-2} Z F+\mathfrak{g}+\mathfrak{f}^{n-1} & \text { if } \alpha_{i}>\alpha_{j} .\end{cases}
\end{aligned}
$$

Proof. We have

$$
\frac{\partial}{\partial x_{k}}(\mathfrak{g}) \subseteq \mathfrak{S}+p^{\alpha}{ }^{k} Z F ; \frac{\partial}{\partial x_{k}}\left(\mathfrak{f}^{n}\right) \subseteq \mathfrak{f}^{n-1}
$$

Thus since $d_{i j} \equiv t\left(x_{i}\right) a_{i j} \bmod \left(\mathfrak{g}+\tilde{f}^{n}\right)$ with $a_{i j} \in Z$, it follows that

$$
\frac{\partial}{\partial x_{k}} d_{i j} \equiv 0 \bmod \left(p^{\alpha_{k}} Z F+\mathfrak{g}+\mathfrak{f}^{n-1}\right) \text {. }
$$

By (4) and $d_{i j} \equiv t\left(x_{i}\right) a_{i j} \bmod \left(\mathfrak{g}+\tilde{f}^{n}\right)$, we have

$$
\frac{\partial}{\partial x_{i}} d_{i j} \equiv a_{i j}\left(\begin{array}{c}
p^{\alpha_{i}} \\
p
\end{array}\right)(p-1)\left(x_{i}-1\right)^{p-2} \bmod \left(p^{\alpha_{i}} Z F+\mathfrak{j}+\mathfrak{f}^{n-1}\right) \text {. }
$$

Since $p^{\alpha_{i}-1}$ divides $\left(\begin{array}{c}p^{\alpha_{i}} \\ p\end{array}\right), \frac{\partial}{\partial x_{i}} d_{i j} \equiv 0 \bmod \left(p^{\alpha_{i}-1}\left(x_{i}-1\right)^{p-2} Z F+p^{\alpha_{i}} Z F+\right.$ $\left.\xi+\tilde{f}^{n-1}\right)$. If $\alpha_{i}=\alpha_{j}$ then $b_{i j} \in Z$, and we may differentiate $d_{i j} \equiv t\left(x_{j}\right) b_{i j}$ with 
respect to $x_{i}$ to obtain the desired result.

Next, we need to expand $\left[x_{i}, x_{j}\right]^{d_{\imath j}}-1$ modulo $\left(\mathfrak{f}^{2} \xi+\mathfrak{f}^{n+2}\right)$. We first observe,

$$
\begin{aligned}
& {\left[x_{i}, x_{j}\right]_{i}^{\beta_{i}} x_{i+1}^{\beta_{i+1}} \cdots x_{m}^{\beta_{m}}-1} \\
& \quad \equiv x_{m}^{-\beta_{m}} \cdots x_{i+1}^{\beta_{i+1}} x_{i}^{-\beta_{i}}\left(\left[x_{i}, x_{i}\right]-1\right) x_{i}^{\beta_{i}} x_{i+1}^{\beta_{i}+1} \cdots x_{m}^{\beta_{m}} \\
& \quad \equiv\left(\left[x_{i}, x_{j}\right]-1\right) x_{i}^{\beta_{i}} x_{i+1}^{\beta_{i+1}} \cdots x_{m}^{\beta_{m}}-\sum_{k=i}^{m} \beta_{k}\left(x_{k}-1\right)\left(\left[x_{i}, x_{i}\right]-1\right) x_{i}^{\beta_{i}} x_{i+1}^{\beta_{i}+1} \cdots x_{m}^{\beta_{m}} \\
& \quad \equiv\left(\left[x_{i}, x_{j}\right]-1\right) x_{i}^{\beta_{i}} \cdots x_{m}^{\beta_{m}}-\sum_{k=i}^{m}\left(x_{k}-1\right)\left(\left[x_{i}, x_{j}\right]^{x_{h}\left(\partial / \partial x_{k}\right)\left(x_{i}^{\beta_{i}} \cdots x_{m}^{\beta_{m}}\right)}-1\right) .
\end{aligned}
$$

Thus,

$$
\left[x_{i}, x_{j}\right]^{d_{i j}}-1 \equiv\left(\left[x_{i}, x_{j}\right]-1\right) d_{i j}-\sum_{k=i}^{m}\left(x_{k}-1\right)\left(\left[x_{i}, x_{j}\right]^{x_{k}\left(\partial / \partial x_{k}\right) d_{i j}}-1\right) .
$$

Now, modulo $\left(\mathfrak{f}^{2} \precsim+\mathfrak{f}^{n+2}\right)$

$$
\begin{aligned}
\left(\left[x_{i}, x_{j}\right]-1\right) d_{i j} \equiv & x_{i}^{-1} x_{j}^{-1}\left\{\left(x_{i}-1\right)\left(x_{j}-1\right)-\left(x_{j}-1\right)\left(x_{i}-1\right)\right\} d_{i j} \\
\equiv & \left\{\left(x_{i}-1\right)\left(x_{j}-1\right)-\left(x_{j}-1\right)\left(x_{i}-1\right)\right\} d_{i j} \\
& \quad-\left(x_{i}-1\right)\left\{\left(x_{i}-1\right)\left(x_{j}-1\right)-\left(x_{j}-1\right)\left(x_{i}-1\right)\right\} d_{i j} \\
& \quad-\left(x_{j}-1\right)\left\{\left(x_{i}-1\right)\left(x_{j}-1\right)-\left(x_{j}-1\right)\left(x_{i}-1\right)\right\} d_{i j} \\
\equiv & \left(x_{i}-1\right)\left(x_{j}-1\right) d_{i j}-\left(x_{j}-1\right)\left(x_{i}-1\right) d_{i j}, \\
\quad \text { by }(6) \text { and }(8) & \left(x_{i}-1\right)\left(x_{j}-1\right) t\left(x_{j}\right) b_{i j}-\left(x_{j}-1\right)\left(x_{i}-1\right) t\left(x_{i}\right) a_{i j} \\
\equiv & \quad \text { by Lemma } 2.1 \\
\equiv & \left(x_{i}-1\right)\left(x_{j}^{p^{\alpha} b_{i j}}-1\right)-\left(x_{i}-1\right)\left(x_{i}^{p^{\alpha_{i}}}-1\right)
\end{aligned}
$$

Thus we have,

Lemma 2.3. Modulo $\left(\mathfrak{f}^{2} \mathfrak{g}+\mathfrak{f}^{n+2}\right)$,

$$
\begin{aligned}
{\left[x_{i}, x_{j}\right]^{d_{i j}}-1 \equiv } & \left(x_{i}-1\right)\left(x_{j}^{p^{\alpha} j b_{i j}}-1\right)-\left(x_{j}-1\right)\left(x_{i}^{p^{\alpha} a_{i j}}-1\right) \\
& -\sum_{k=i}^{m}\left(x_{k}-1\right)\left(\left[x_{i}, x_{j}\right]^{x_{k}\left(\partial / \partial x_{k}\right) d_{i j}}-1\right) .
\end{aligned}
$$

Finally, using (6) and (8), we have, for any $x_{k}, \bmod \left[F^{\prime}, S\right] \gamma_{n+3}(F)$,

$$
\begin{aligned}
{\left[\left[x_{i}, x_{j}\right]^{d_{i j}}, x_{k}\right] } & \equiv\left[x_{i}, x_{j}, x_{k}\right]^{d_{i j}} \\
& \equiv\left[x_{i}, x_{k}, x_{j}\right]^{d_{i j}}\left[x_{k}, x_{i}, x_{i}\right]^{d_{i j}} \\
& \equiv\left[x_{i}, x_{k}\right]^{\left(-1+x_{j}\right) d_{i j}}\left[x_{k}, x_{j}\right]^{\left(-1+x_{i}\right) d_{i j}} \\
& \equiv 1 .
\end{aligned}
$$

Thus we have, 
Lemma 2.4 (Gupta [2]). $\left[D_{n+2}(\mathfrak{f} \mathfrak{E}), F\right] \subseteq\left[F^{\prime},{ }_{-} S\right] \gamma_{n+3}(F)$ for all $n \geqq 0$.

This completes our preliminary discussions.

\section{§3. The main theorem}

Let $G$ be a finitely generated metabelian $p$-group. Then $G$ admits a presentation of the form

$$
G=F / R=\left\langle x_{1}, x_{2}, \cdots, x_{m} ; x_{1}^{\gamma^{\alpha_{1}}} \zeta_{1}, x_{2}^{p^{\alpha_{2}}} \zeta_{2}, \cdots, x_{m}^{p^{\alpha_{m}}} \zeta_{m}, \zeta_{m+1}, \cdots, F^{\prime \prime}\right\rangle,
$$

where $\alpha_{1} \geqq \alpha_{2} \geqq \cdots \geqq \alpha_{m}>0$ (see for instance [4], page 149). Let $S$ be the normal subgroup of $F$ generated by $x_{1}^{p \alpha_{1}}, x_{2}^{p \alpha_{2}}, \cdots, x_{m}^{p \alpha_{m}}$ and $F^{\prime}$, then it follows that $S^{\prime} \subseteq R \subseteq S$. In terms of the free group rings, the dimension subgroup $D_{n+2}(G)=D_{n+2}(\mathfrak{x}) / R$, where $\mathfrak{x}=Z F(R-1)$ and $D_{n+2}(\mathfrak{x})=F \cap$ $\left(1+\mathfrak{r}+\mathfrak{f}^{n+2}\right)$. Then $R r_{n+2}(F) \subseteq D_{n+2}(\mathfrak{r})$. If $z \in D_{n+2}(\mathfrak{x})$, then $z-1 \in \mathfrak{x}+\mathfrak{f}^{n+2}$ implies that $z r-1 \in \mathfrak{f r}+\mathfrak{f}^{n+2}$ for some $r \in R$. It follows that $D_{n+2}(G)=$ $\gamma_{n+2}(G)$ if and only if $D_{n+2}(\mathfrak{f r})=F \cap\left(1+\mathfrak{f r}+\mathfrak{f}^{n+2}\right) \subseteq R \gamma_{n+2}(F)$. We now prove our main result.

Theorem 3.1. $\quad D_{n+2}^{2}(\tilde{f} \mathfrak{r}) \subseteq R \gamma_{n+2}(F)$ for all $n \leqq p$.

Proof. Let $w \in D_{n+2}(\mathfrak{f} x)$. Then $w-1 \in \mathfrak{f r}+\mathfrak{f}^{n+2} \subseteq \mathfrak{f} \xi+\mathfrak{f}^{n+2}$, and by Lemma 2.1,

$$
w \equiv \prod_{1 \leqq i<j \leqq m}\left[x_{i}, x_{j}\right]^{d_{i j}} \bmod F^{\prime \prime},
$$

where $d_{i j} \equiv t\left(x_{i}\right) a_{i j} \equiv t\left(x_{j}\right) b_{i j} \bmod \left(\mathfrak{g}+\mathfrak{f}^{n}\right)$. Now, $w-1 \in \mathfrak{F} \mathfrak{r}+\mathfrak{f}^{n+2}$ implies $w-1 \in \mathfrak{f} \mathfrak{x}+\mathfrak{f}^{2} \mathfrak{g}+\mathfrak{f}^{n+2}$. Then it follows by Lemma 2.3, that

$$
w-1 \equiv \sum_{k=1}^{m}\left(x_{k}-1\right)\left(y_{k} u_{k}^{-1}-1\right) \equiv 0 \bmod \left(\mathfrak{f} \mathfrak{r}+\mathfrak{f}^{2} \mathfrak{g}+\mathfrak{f}^{n+2}\right),
$$

where

$$
y_{k}=\prod_{i<k} x_{i}^{-p^{\alpha} i a_{i k}} \prod_{k<j} x_{j}^{p^{\alpha j b} j k}, \quad u_{k}=\prod_{\substack{i<j \\ i \leqq k}}\left[x_{i}, x_{j}\right]^{x_{k}\left(\partial / \partial x_{k}\right) \alpha_{\imath j}} .
$$

From (10) it follows that for each $k=1,2, \cdots, m$,

$$
y_{k} u_{k}^{-1}-1 \in \mathfrak{x}+\mathfrak{f} \mathfrak{g}+\mathfrak{f}^{n+1},
$$

which yields, in turn, using $\mathfrak{f r} \subseteq \mathfrak{f} \xi$,

$$
y_{k} u_{k}^{-1} r_{k}-1 \in \mathfrak{f} g+\mathfrak{f}^{n+1}
$$

with some $r_{k} \in R$, and by Lemma 2.4, for all $k=1,2, \cdots, m$, 


$$
\left[x_{k}, y_{k} u_{k}^{-1} r_{k}\right] \in R \gamma_{n+2}(F) \text {, }
$$

which reduces to

$$
\left[x_{k}, y_{k} u_{k}^{-1}\right] \in R r_{n+2}(F)
$$

and hence

$$
\left[x_{k}, u_{k}^{-1}\right]\left[x_{k}, y_{k}\right] \in R r_{n+2}(F) .
$$

Next, $\left[x_{k}, u_{k}^{-1}\right] \equiv\left[x_{k}, u_{k}\right]^{-1} \bmod R \gamma_{n+2}(F)$, and $\left[x_{k}, u_{k}\right]$ is a product of commutators of the form

$$
\left[x_{k},\left[x_{i}, x_{j}\right]^{x_{k}\left(\partial / \partial x_{k}\right) d_{i j}}\right], \quad 1 \leqq i \leqq k, \quad 1 \leqq i<j \leqq m .
$$

By Lemma 2.2, for either $i<k$ or $i=k$ and $\alpha_{i}=\alpha_{j}$,

$$
\begin{aligned}
{\left[x_{k},\left[x_{i}, x_{j}\right]^{x_{k\left(\partial / \partial x_{k}\right) d_{i j}}}\right] } & \equiv\left[x_{k},\left[x_{i}, x_{j}\right]^{\alpha_{k x_{k} v}}\right] \text { for some } v \in Z F \\
& \equiv\left[x_{k}^{p^{\alpha_{k}}},\left[x_{i}, x_{i}\right]^{x_{k} v}\right] \\
& \equiv 1 \bmod \left[F^{\prime}, S\right] \gamma_{n+2}(F)
\end{aligned}
$$

If $i=k$ and $\alpha_{i}>\alpha_{j}$, then by Lemma 2.2, for some $v, w \in Z F$,

$$
\begin{aligned}
& {\left[x_{i},\left[x_{i}, x_{j}\right]^{x_{i}\left(\partial / \partial x_{i}\right) d_{i j}}\right] \equiv\left[x_{i},\left[x_{i}, x_{j}\right]^{x_{i} p^{\alpha_{i v+p^{\alpha}} \alpha^{-1}\left(x_{i}-1\right)}{ }^{p-2} w}\right]} \\
& \equiv\left[\left[x_{i}, x_{j}\right]^{\left(x_{i}-1\right)^{p-2} \cdot p^{\alpha_{i}-1} w}, x_{i}\right]^{-1} \\
& \equiv[x_{j}^{p^{\alpha_{j}}}, \underbrace{x_{i}, \cdots, x_{i}}_{p}]^{p^{\alpha_{i}-1-\alpha_{j w}}} \bmod \left[F^{\prime}, S\right] \gamma_{n+2}(F) \\
& \equiv[\zeta_{j}, \underbrace{x_{i}, \cdots, x_{i}}_{p}]^{\alpha_{i}-1-\alpha_{j} w} \bmod R \gamma_{n+2}(F) \\
& \equiv 1 \bmod R r_{n+2}(F) \text {. }
\end{aligned}
$$

Thus (11) is reduced to $\left[x_{k}, y_{k}\right] \in R r_{n+2}(F)$. However,

$$
\begin{aligned}
{\left[x_{k}, y_{k}\right] } & \equiv \prod_{i<k}\left[x_{i}^{p^{\alpha} i a_{i k}}, x_{k}\right] \prod_{k<j}\left[x_{k}, x_{j}^{p^{\alpha} b_{k j}}\right] \\
& \equiv \prod_{i<k}\left[x_{i}, x_{k}\right]^{d_{i k}} \prod_{k<j}\left[x_{k}, x_{j}\right]^{d_{k j}} \bmod \left[F^{\prime}, S\right] \gamma_{n+2}(F) .
\end{aligned}
$$

Thus

$$
w^{2} \equiv \prod_{k=1}^{m}\left[x_{k}, y_{k}\right] \equiv 1 \bmod R \gamma_{n+2}(F)
$$

This completes the proof of our main theorem.

As a corollary we obtain,

TheOREM 3.2. Let $G$ be a finitely generated metabelian p-group. Then 
(a) $D_{n+2}(G)=r_{n+2}(G)$ for all $n \leqq p-1$,

(b) if $p=2, D_{4}^{2}(G) \subseteq \gamma_{4}(G)$,

(c) if $p$ is odd, $D_{p+2}(G)=\gamma_{p+2}(G)$.

For $p=3$, part (a) of Theorem 3.2 was first proved by Passi [6]; part (b) is due to Losey [3]. We refer the reader to Passi [7] for a general background on the dimension subgroup problem.

\title{
REFERENCES
}

[1] N. Gupta, On the dimension subgroups of metabelian groups, J. Pure Appl. Algebra, 24 (1982), 1-6.

[2] - Sjogren's Theorem for dimension subgroups-The metabelian case, Annals of Math. Study (1985), to appear.

[ 3 ] G. Losey, N-series and filtration of the augmentation ideal, Canad. J. Math., 26 (1974), 962-977.

[4] W. Magnus, A. Karrass and D. Solitar, Combinatorial Group Theory, Interscience, New York, 1966.

[5] S. Moran, Dimension subgroups mod n, Proc. Camb. Phil. Soc., 68 (1970), 579582.

[6 ] I.B.S. Passi, Dimension subgroups, J. Algebra, 9 (1968), 152-182.

[7] - Group Rings and Their Augmentation Ideals, Springer Lecture Notes in Math., 715 (1979), Springer-Verlag, Berlin-Heidelberg-New York.

[ 8 ] E. Rips, On the fourth integer dimension subgroup, Israel J. Math., 12 (1972), $342-346$.

[ 9 ] J. A. Sjogren, Dimension and lower central subgroups, J. Pure Appl. Algebra, 14 (1979), 175-194.

[10] K. Tahara, On the structure of $Q_{3}(G)$ and the fourth dimension subgroup, Japan. J. Math. (New Ser.), 3 (1977), 381-394.

[11] - The fourth dimension subgroups and polynomial maps, II, Nagoya Math. J., 69 (1978), 1-7.

\author{
Narain Gupta \\ Department of Mathematics \\ University of Manitoba \\ Winnipeg, R3T 2N2 \\ Canada \\ Ken-Ichi Tahara \\ Department of Mathematics \\ Aichi University of Education \\ Kariya, 448 \\ Japan
}

